\title{
DEVELOPMENT OF GPS SATELLITE VISIBILITY SIMULATION METHOD UNDER URBAN CANYON ENVIRONMENT
}

\author{
Dusik $\mathrm{Kim}^{1 *}$, Junhee Youn ${ }^{1}$, Taehoon $\mathrm{Kim}^{1}$ and Gihong Kim${ }^{2}$ \\ ${ }^{1}$ Korea Institute of Civil Engineering and Building Technology (KICT), 283, Goyangdae-ro, Ilsanseo-gu, Goyang-si, Gyeonggi-do, \\ 10223, Rep. of Korea - (dusikkim, younj, kth)@kict.re.kr \\ ${ }^{2}$ Gangneung-Wonju National University, 7, Jukheon-gil, Gangneung-si, Gangwon-do, 25457, Rep. of Korea - ghkim@gwnu.ac.kr
}

KEY WORDS: UAV, Urban Positioning, GPS, Simulation, Multipath

\begin{abstract}
:
When the UAV is flying in a lower area than the buildings in the urban environment, the GPS positioning accuracy of the aircraft reduced due to the blocked or reflected signals. Therefore, when the UAVs fly in this area, it should be able to provide a criterion that can give warning message to the UAV users such as stop the auto-flight mode or not rely too much on the position of the UAV represented on the monitor of GCS. In this study, the Elevation Mask Profile (EMP) generation algorithm proposed which calculates azimuth and elevation angle ranges blocked by obstacles at certain grid points by using DSM. It is a core algorithm for a GPS simulator developed that predict the blocked satellite signal by matching the EMP calculated for each grid point with the GPS satellites' azimuth and elevation angle information at the grid point. According to the comparison of EMP and observed data, most of the results had correlation with obstacles nearby. We found some signal blocking areas were not determined as EMP due to the skyscrapers located out of the target area where DSM was constructed. To overcome this limitation, larger spatial data than the DSM that used in this study must be applied. Furthermore, additional studies about optimal search radius definition in EMP calculation procedure to acquire more accurate and standardized EMP are necessary.
\end{abstract}

\section{INTRODUCTION}

UAV technology has been propagated in various application fields such as leisure, broadcasting, monitoring (i.e. for fire, facilities, and crops), logistics, first aid and aid delivery, fire detection, precision agriculture, and 3D mapping due to the characteristics of information obtained from various perspectives and through rapid mobility. It is expected that UAVs in the future will perform an endless array of diverse missions depending on the combination of sensors and features mounted on their platforms. In particular, the introduction of UAV technology in fields where involvements of aircrafts were difficult due to the economic efficiency and mission duration is expected to be carried out (Youn et al., 2016).

We should prepare the age of millions of UAVs operating at the same time in the near future. When a number of the UAVs fly without rules, there can be a risk of crash with other UAVs, the ground obstacles, and aircrafts and it can cause casualty and property damage by the crash secondarily. Therefore, safety issues must be resolved primarily in order to activate the UAV markets and the UAV traffic management system has emerged as a vital importance for safety issues.

As UAVs are used in low altitudes usually unlike air traffic management system reflecting 3D spatial information to prevent collisions with the ground structures should be developed. Although obstacle avoidance techniques utilizing UAV's ultrasonic (Gageik et al., 2015) or optical (Hrabar et al., 2005) sensors are also being developed, the pathway of the UAV should be built to reduce the probability of accidents significantly. To this end, we are pushing ahead the convergence of 3D spatial information to the future UAV traffic management (UTM) system (MOLIT, 2016; KAIA, 2017). We are planning to develop a UTM based on 3D grid system as a technological breakthrough of this issue (Youn et al., 2018).

Radišić et al. (2018) pointed out that the UAV application field in the urban area can affect the surveillance and navigation of the UAV due to the environmental characteristics of the obstacles. Especially in GPS positioning, it is analysed that multipath signals of GPS, which occur frequently in urban areas, cause a decrease in positioning accuracy. Zimmermann et al. (2017) announced that it is possible to improve UAV positioning accuracy through shadow matching technology using 3D building model to ensure positioning accuracy in mapping using UAV. The recent publication of these studies suggests that there is a need to improve UAV position accuracy.

When the UAV is flying in a lower area than the buildings in the urban canyon environment, the GPS positioning result of the aircraft can be reduced due to the decrease of the visible satellite or the multipath signal. Therefore, when the UAV enters an area where the position accuracy can be degraded, it should be able to stop the automatic flight mode, or provide a criterion that can warn that the UAV does not rely too much on the position of the UAV represented on the GCS (Ground Control System) monitor.

In this study, we proposed the Elevation Mask Profile (EMP) generation algorithm that calculates azimuth and altitude angles that can be shadowed by obstacles at certain grid intervals on the road using DSM (Digital Surface Model). We also proposed a simulator that can predict the shielded satellite signal by matching the EMP calculated for each grid point with the azimuth angle information of the satellite based on the location of the points. In addition, the expected effect of EMP was analysed through the data acquired using actual GPS receiver.

\footnotetext{
* Corresponding author
} 


\section{DEVELOPMENT OF EMP GENERATION ALGORITHM}

One of the popular simulation method of the GPS satellite observation environment is line-polygon collision checking (Akenine-Moller et al., 1999). However, since this method requires $3 \mathrm{D}$ vector information, it is difficult to identify the complicated geometric relationship between user location, satellite position, and obstacles' information. Generally, when $3 \mathrm{D}$ vector information is used for visibility analysis, the $3 \mathrm{D}$ building model is used, or 3D altitude information is substituted into 2D information of the building area in the digital map. However, when these data are utilized in visibility analysis, there is a problem that it does not reflect all objects in the real world such as trees and utility poles.

Recently, the development of mapping technology that using UAVs has popularized, so UAV users can construct geospatial information such as aerial orthoimage, point clouds of terrain objects, and DSM for a small area. In this study, we proposed an algorithm that simulate the observation environment of the navigation satellite in the flyable area based on the DSM constructed from the image data acquired from the UAV. Using this method, the signal-shadowed area due to the surrounding obstacles is determined in advance at a specific target point in the $3 \mathrm{D}$ space. If this signal-shadowed area database already constructed, UAVs can identify the actual visible satellite. In addition, UAVs can recognize regions that are difficult to receive GPS signals through the signal-shadowed area database, and they can distinguish multipath signals. In this study, the area where signals are blocked by surrounding obstacles is called the Elevation Mask Profile (EMP). The implementation sequence of the EMP generation algorithm is shown as Figure 1.

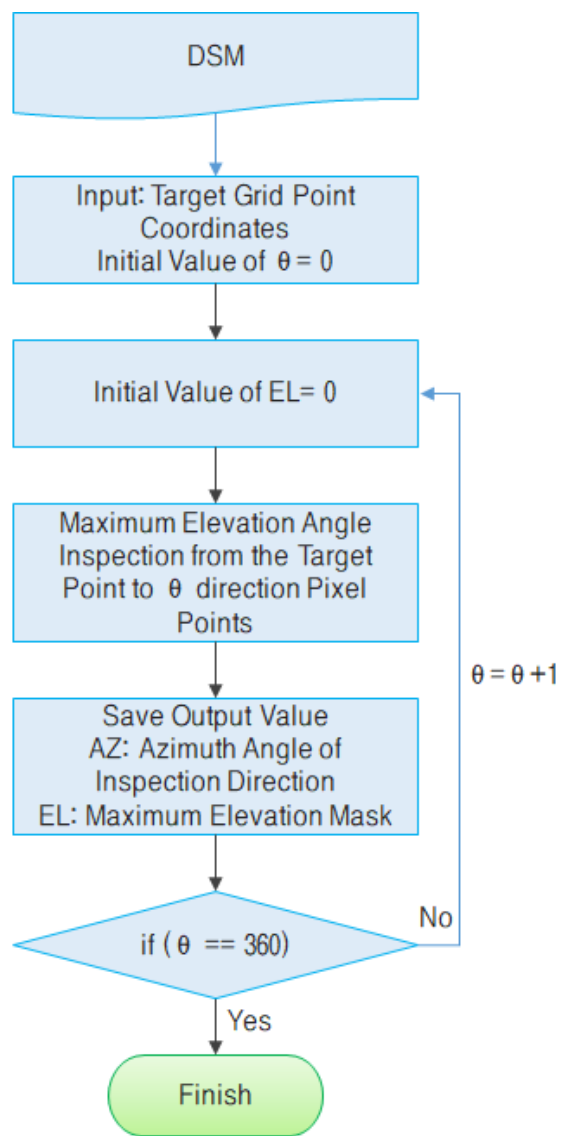

Figure 1. Flow chart of EMP generation algorithm
In order to develop and verify the EMP generation algorithm, we used the DSM of the Korea Institute of Civil Engineering and Building Technology (KICT). The DSM was generated based on the point-cloud data, which was acquired by UAV photogrammetry. Figure 2 shows the example of constructed 3D model of KICT by using this UAV data.

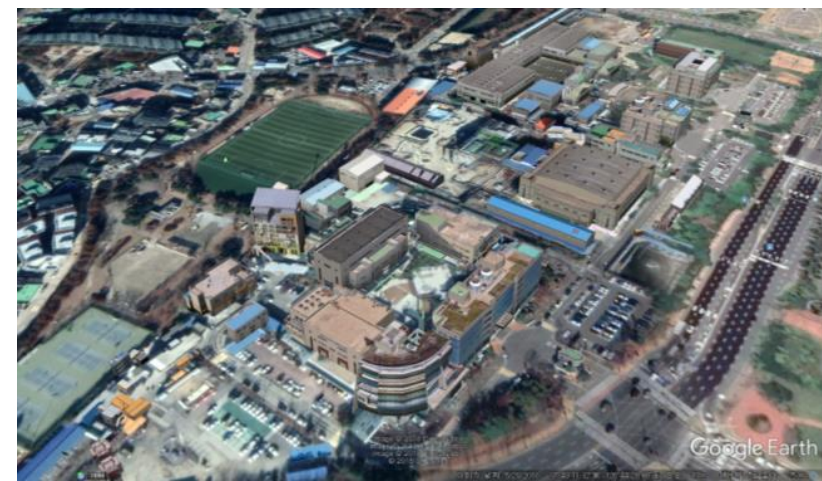

Figure 2. 3D model of KICT

Here, the spatial resolution of the DSM is $5 \mathrm{~cm} /$ pixel, and the upper end of the image is in the north direction. The coordinate system of the DSM was Korea TM (Transverse Mercator), plane rectangular coordinate system in Korea. We generated two layers, which describe buildings and roads, for effective visualization of the result of EMP generation algorithm. To generate these two layers, we used orthoimage of the KICT, which has same spatial resolution as DSM. Figure 3 shows the layers created by extracting the building area and road area from the original DSM in the target area. The blue pixels represent the building area, and the red pixels represent the road area.

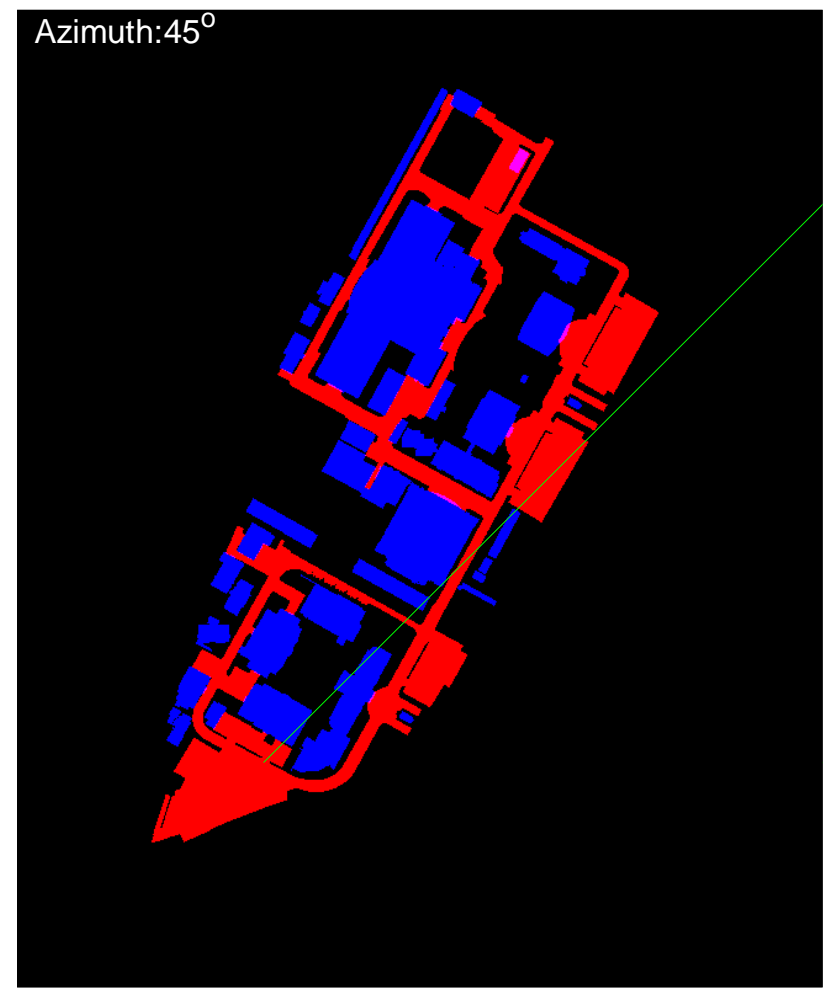

Figure 3. Example of EM calculation process

Figure 4 shows the detail of example. As shown in Figure 4, the EMP generation algorithm implements a straight-line equation for a specific azimuth angle from an initial target point to the 
end of image. Then, the angle of the signal shadowed elevation angle by each pixel was calculated by using the altitude difference and the distance between the target point and the pixel position with respect to the pixels passing through this straight line. Finally, the maximum value among the calculated signal shadowed elevation angle for each pixel was determined as the elevation mask (EM) for the given azimuth angle. The green straight line in Figure 3 shows the result of implementing a straight line corresponding to the $45^{\circ}$ azimuth angle when the checkpoint is set to the southwest of the target area. This explains the EM calculation process for one azimuth. If iteration is performed while changing theta, EMP of the given target point can be generated for all azimuth directions.

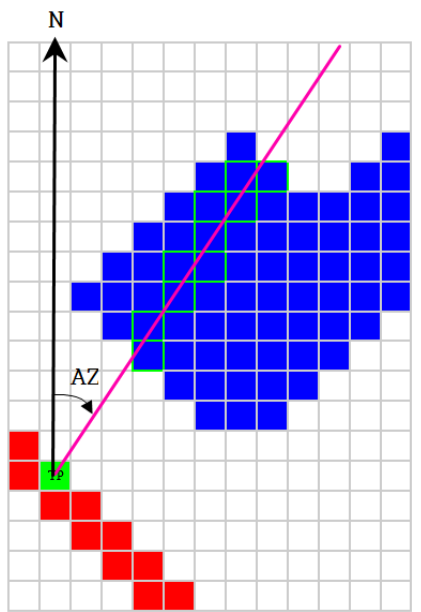

Figure 4. Detailed process of EMP generation algorithm

Figure 5 shows the sky-plot of the calculated EMP $\left(1^{\circ}\right.$ interval for all azimuth angle) with respect to target point. In the Figure 5 , the red line represents the maximum elevation angle at which the navigation satellite signal can be shadowed. For example, a range between $120^{\circ}$ and $270^{\circ}$ of azimuth showed zero EMPs because the target point was selected to the south of the image and there is no obstacles in south directions of the target point. On the other hand, relatively higher EMPs were calculated for the north azimuth. The geographical characteristics of the south outside of target area is open terrain environment, however there are some obstacles, those can cause signal blocking, far away from target area. Therefore, in order to apply this algorithm to actual service, using a larger 3D model than this study recommended improving algorithm accuracy.

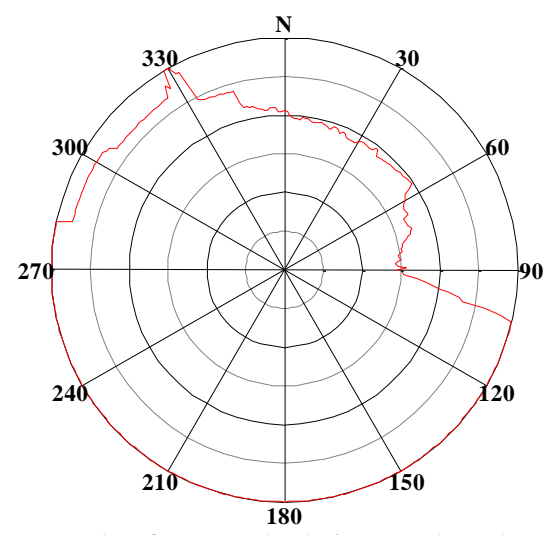

Figure 5. Example of EMP calculation result at the target point in Figure 3

\section{FIELD TEST AND DATA ANALYSIS}

In order to evaluate the EMP generation algorithm, we checked how GPS signals are received at specific points in the actual target area. The experiment was carried out at the green point in the left map of Figure 6 so that the signal block effect by the obstacles can be seen much. As shown in landscape pictures (Figure 7), the southeast and northeast directions are composed of some open terrain sections. In the case of northwest and northeast, most of the satellite signals will blocked by neighbouring buildings.

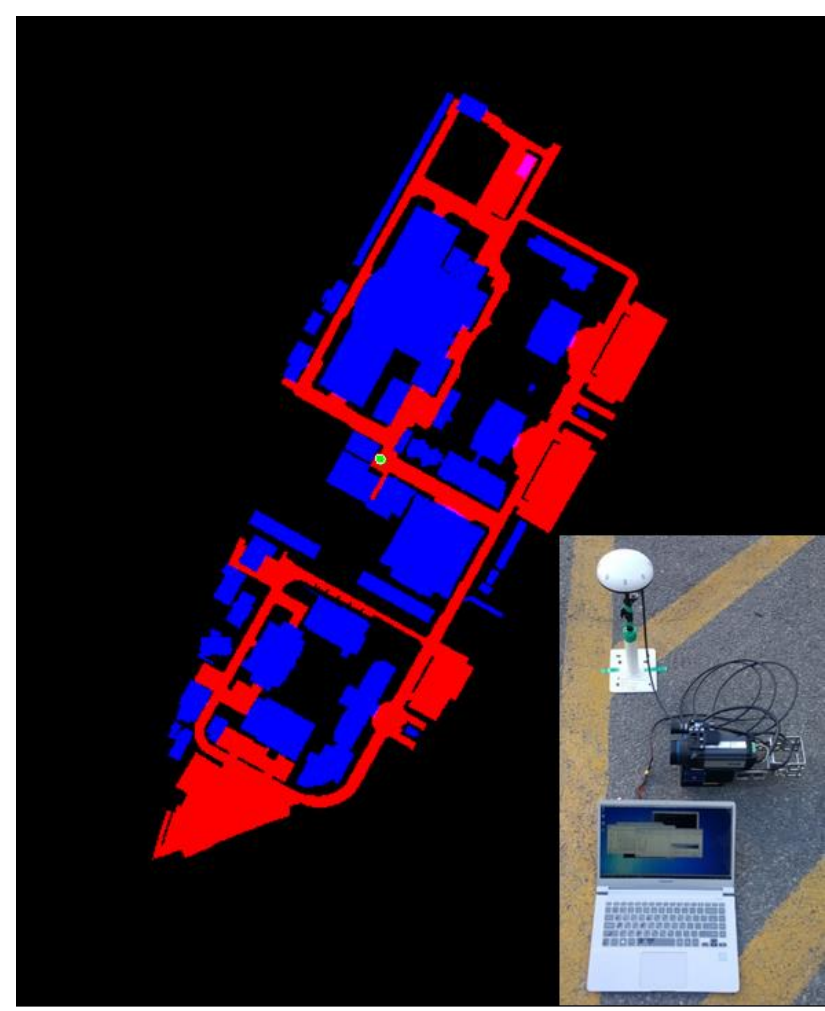

Figure 6. Field test position and RTK GPS positioning
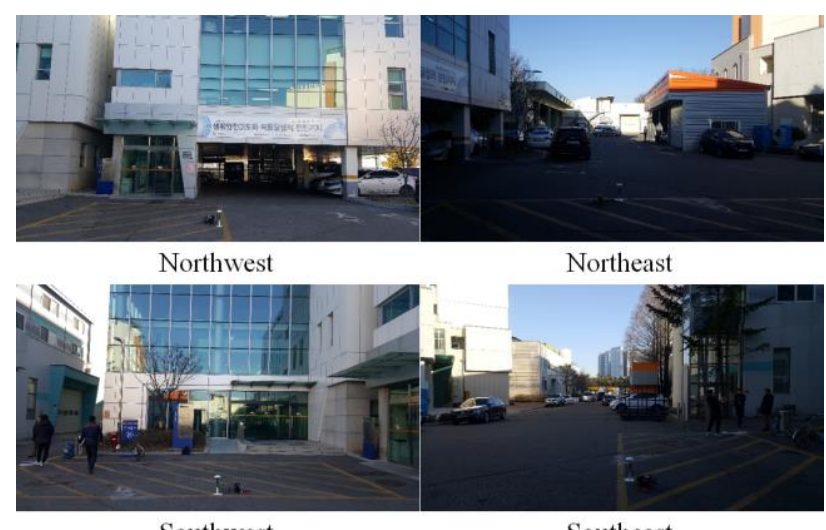

Southwest

Southeast

Figure 7. Landscape for each direction of target point

The test was held about 20 minutes, and we determined the reference coordinate of target point by averaging network RTK results, which consist of 1 -second intervals. In addition, we recorded the raw measurements of GPS as RINEX observation during the same time. Then, we processed the observation file by using RTKlib (Takasu, 2009) as static positioning mode. For the data processing, we used broadcast orbit downloaded from NASA FTP server (ftp://cddis.gsfc.nasa.gov). 
After data processing, we analysed the information about GPS satellite azimuth, elevation angle and L1 multipath by merging the satellite information and EMP as shown in Figure 8. In the sky-plot of Figure 8, the red line represents the EMP and the points represent the GPS satellite trajectory. Depending on the intensity of the L1 multipath, the colors of the points are implemented in the order of red, yellow, green, and blue. That is, L1 multipath is weak in G25 and G32 satellites, and stronger in G31 than G25th and G32 satellites. As shown in Figure 8, it is confirmed that the satellite located in the open-sky does not receive the influence of the multipath as compared with the rest of the satellites.

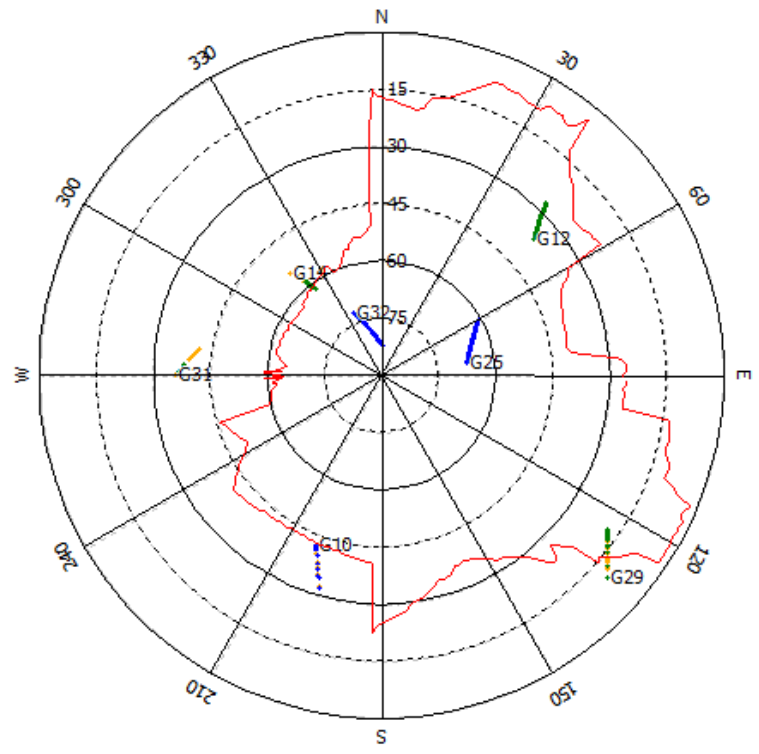

Figure 8. Result of field test

Figure 9 shows the sky-plot of GPS satellites' signal strength and 3D model of urban test area (Kim et al., 2015). The test took about 4 hours on the sidewalk in Teheran-Ro, Gangnam$\mathrm{Gu}$, Seoul. Teheran-Ro is a typical urban environment crowded with skyscrapers. When vehicles enter the Teheran-Ro, the number of visible GPS satellites sharply decrease and the accuracy of the positioning deteriorates frequently. In the skyplot of Figure 9, the point represents the trajectory of all observed GPS satellites, and the color of each point is configured as a legend according to the $\mathrm{S} 1$ value of the GPS observation RINEX file. The background layer beneath the skyplot represents the result of captured 3D model from V-World, the 3D spatial information service of South Korea (http://map.vworld.kr). The center of sky-plot and the center of the image is consistent with the observation point.

In the urban environment, multipath GPS signals can be recorded in the observation data, even if there are obstacles between direct path of the GPS satellites and reciever as shown in Figure 9. Moreover, these multipath signals will significantly reduce the positioning accuracy. The S1 values are clearly low in the direction, which obscured by the buildings, however it is difficult to determine whether this is a multipath signal or not through a threshold value. Therefore, applying the EMP generation algorithm proposed in this study will provide a reliable reference for eliminating multipath signals at observation points.

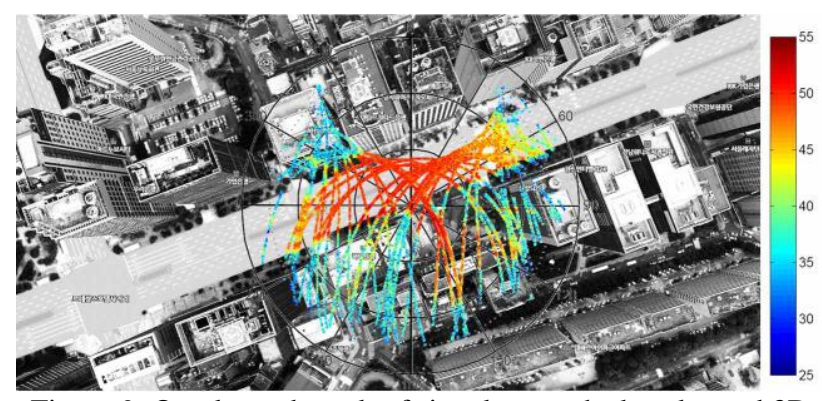

Figure 9. Overlapped result of signal strength sky-plot and 3D model (Kim et al., 2015)

\section{CONCLUSIONS}

We proposed the EMP generation algorithm that calculates azimuth and altitude angles that can be shadowed by obstacles at certain grid intervals on the road using DSM. This algorithm is based on raster data processing such as image processing. Therefore, this algorithm can be applied wherever the DSM exists.

Recently, digital twin technology, which is a link between the real world and the cyber world, is proposed as a solution for the implementation of smart city. However, it seems that current computing power will not be able to handle with the realization of the complex real world through the cyber world. 3D grids can be used as an alternative to overcome these limitations.

In this study, we developed EMP generation algorithm based on DSM, which is 3D grid data. As a result, it was possible to quickly determine the signal blocking element around the point by inputting a specific point. In the future, we will be able to utilize it in low altitude UAV or autonomous vehicle by integrating the simulation technology with the satellite orbit and constructing a data calculation / provision system.

\section{ACKNOWLEDGEMENTS}

This research, 'Development of Integrated Land Management Support Technology Based on 3 dimensional Geo-Spatial grid system', was supported by the Ministry of Land, Infrastructure and Transport (MOLIT) of Korea, under the national spatial information research program supervised by the Korea Agency for Infrastructure Technology Advancement (KAIA) (19NSIPC135506-03)

\section{REFERENCES}

Akenine-Moller, T., Haines, E. and Hoffman, N., 2018. Realtime rendering, AK Peters/CRC Press.

Gageik, N., Benz, P. and Montenegro, S., 2015. Obstacle detection and collision avoidance for a UAV with complementary low-cost sensors. IEEE Access, Vol. 3, pp. 599609.

Hrabar, S., Sukhatme, G. S., Corke, P., Usher, K., and Roberts, J., 2005. Combined optic-flow and stereo-based navigation of urban canyons for a UAV. 2005 IEEE/RSJ International Conference on Intelligent Robots and Systems, pp. 3309-3316.

KAIA, 2017. Final Report for $1^{\text {st }}$ Year of Research Project: Development of Integrated Land Management Support 
Technology Based on 3 dimensional Geo-Spatial grid system Research.

Kim, D., Park, K.-D., Kim, H.-I., Tae, H., 2015. A Study on Analysis of Multipath Signal Detection using GPS Signal Strength Information. Journal of Navigation and Port Research, Vol. 39, No. 1, pp. 7-13.

MOLIT, 2016. Pilot 3D Spatial Information Construction for Drone Roads, Ministry of Land, Infrastructure and Transport. Radišić, T., Vidović, A., Ivošević, J., Wang, B., 2018. Challenges and Solutions for Urban UAV Operations. International Scientific Conference "Science and Traffic Development" (ZIRP 2018), pp. 1-11.

Takasu, T., 2009. RTKLIB: Open Source Program Package for RTK-GPS, FOSS4G 2009 Tokyo, Japan.

Youn, J., Kim, C. and Kim, D., 2016. Drone technologies in the construction sector and future strategy for the promotion of the drone industries. Smart City. Korea Institute of Civil Engineering and Building Technology, pp. 198-201.

Youn, J., Kim, D., Kim, T., Yoo, J. H. and Lee, B. J., 2018. Development of UAV air roads by using 3D grid system. ISPRS TC IV, Delft, Netherlands, pp. 731-735.

Zimmermann, F., Eling, C., Klingbeil, L. and Kuhlmann, H. 2017. Precise positioning of UAVs-dealing with challenging RTK-GPS measurement conditions during automated UAV flights. ISPRS Annals of Photogrammetry, Remote Sensing \& Spatial Information Sciences, Vol. 4, pp. 95-102. 